\title{
Evaluation of certainty and uncertainty for Intuitionistic Fuzzy Sets
}

\author{
Hongmei Wang and Sanghyuk Lee* \\ School of Mechatronics, Changwon National University \\ *Institute for Information and Electronics Research, Inha University
}

\begin{abstract}
Study about fuzzy entropy and similarity measure on intuitionistic fuzzy sets (IFSs) were proposed, and analyzed. Unlike fuzzy set, IFSs contains uncertainty named hesistancy, which is contained in fuzzy membership function itself. Hence, designing fuzzy entropy is not easy because of ununified entropy definition. By considering different fuzzy entropy definitions, fuzzy entropy is designed and discussed their relation. Similarity measure was also presented and verified its usefulness to evaluate degree of similarity.
\end{abstract}

Key Words : intiotionistic fuzzy sets; vague sets; fuzzy entropy; similarity measure

\section{Introduction}

Data uncertainty of fuzzy data has been started from analyzing the fuzzy entropy [1]. Those results were emphasized by designing fuzzy entropy explicitly [2-6]. At the same time, similarity measure was also proposed to compute the degree of similarity between fuzzy sets [7-10]. Obtained fuzzy entropy and similarity measure represent complementary information each other. Hence, the relation was shown and derived similarity measure(entropy) from entropy(similarity measure) in our previous literature [9]. Conventional fuzzy entropy has been studied by numerous researchers [2-6]. Pal and Pal analyzed classical Shannon information entropy; Kosko considered the relationship between distance measure and fuzzy entropy; Liu proposed axiomatic definitions of entropy, distance measures, and similarity measures and discussed the relationships among these three concepts. Methods based on fuzzy numbers enable the simple derivation of similarity measures. However, derived similarity measures are restricted to triangular or trapezoidal membership functions [8]. In contrast, similarity measures based on the distance measure are applicable to general fuzzy membership functions, including non-convex fuzzy membership functions [11].

As a generalization of fuzzy sets, intuitionistic fuzzy sets (IFSs) and vague sets were introduced by Atanassov and Gau and Buehrer, respectively [12-15]. Comparison between IFSs and vague sets was pointed out that two sets are the same by Bustince and Burillo [16]. Object description through IFSs make more realistic, practical and accurate. Hence, fuzzy entropy and similarity construction for IFSs are important to obtain more reliable results. From the definition of IFSs, fuzzy entropy and similarity measure on IFSs have been derived. Proposed fuzzy entropy was considered for not only normal fuzzy membership function but also nonnormal membership

Manuscript received Jun. 29, 2010; revised Aug. 20, 2010;

Accepted Sep. 5, 2010.

* Corresponding Author: Sanghyuk Lee function. Unfortunately, there is not unified fuzzy entropy for IFSs yet. In this study, two fuzzy entropy definitions are considered both. By the dual concept, similarity measure was also obtained through fuzzy entropy results.

\section{Preliminaries}

In this chapter, elementary definition of IFSs, entropy and similarity measure definition fuzzy set/IFSs are defined.

\subsection{Intuitionistic fuzzy sets}

Atanassov gave the definition of IFSs, which contains the uncertainty of data more practically [12].

Definition 2.1 IFSs $V$ in the universe of discourse $X=\left\{x_{1}, x_{2}, \Lambda, x_{n}\right\}$ defined as follows:

$$
\begin{aligned}
V=\left\{\left(x, t_{v}(x), f_{v}(x)\right) \mid x \in X, t_{v}(x) \in[0.1],\right. \\
\left.f_{v}(x) \in[0.1], 0 \leq t_{v}(x)+f_{v}(x) \leq 1\right\}
\end{aligned}
$$

where $t_{v}(x)$ and $f_{v}(x)$ denote a membership function and non-membership function of $x$ to $V$ separately. $t_{v}(x)$ is the lowest bound of membership degree of $x$, and $f_{v}(x)$ is the lowest bound of non-membership degree of of $X$, respectively. From the definition, it is clear that membership degree of IFS $V$ should be restricted in $\left[t_{v}, 1-f_{V}\right]$. Degree of uncertainty can be also defined as $1-t_{v}-f_{V}$. Furthermore, if $t_{v}+f_{V}=1$, then IFS $V$ is considered as fuzzy set. To evaluate the uncertainty or entropy on IFSs, hesitancy information, membership and non membership degree have to be considered. At the same time, similarity measure design needs consideration for definition on IFSs comparison with fuzzy sets case.

\subsection{Fuzzy Entropy and Similarity Measure}

Fuzzy entropy was proposed by De Luca and Termini [6], and the axiomatic definition referred to Shannon's probability entropy. 
Definition $2.2[6]$ A real function $e: \operatorname{FS}(X) \rightarrow R^{+}$is called an entropy on $\mathrm{FS}(\mathrm{X})$ if $e$ has the following properties:

(E1) $e(\tilde{A})=0$, if $\tilde{A}$ is a crisp set.

(E2) $e(\tilde{A})$ assumes a unique maximum if $\mu_{\widetilde{A}}=1 / 2$.

(E3) $e(\widetilde{A}) \leq e(\widetilde{B})$ if $\widetilde{A}$ is crisper than $\widetilde{B}$, that is, if $\mu_{\widetilde{A}} \leq \mu_{\widetilde{B}}$ for $\mu_{\widetilde{B}} \leq 1 / 2$ and $\mu_{\widetilde{A}} \geq \mu_{\widetilde{B}}$ for $\mu_{\widetilde{B}} \geq 1 / 2$.

(E4) $e(\tilde{A})=e\left(\tilde{A}^{C}\right)$ where $\tilde{A}^{C}$ is the complement of $\tilde{A}$.

As dual concept with entropy, similarity measure for fuzzy set was suggested by Liu [4]. Liu's definition of similarity measure was based on the distance measure of membership value.

Definition 2.3 [4] Similarity measure for $\forall A, B \in F(X)$ and $\forall D \in P(X)$ has four properties, which are as follows:

(S1) $s(A, B)=s(B, A), \quad \forall A, B \in F(X)$,

(S2) $s\left(D, D^{C}\right)=0, \forall D \in P(X)$,

(S3) $s(C, C)=\max _{A, B \in F(X)} s(A, B), \forall C \in F(X)$,

(S4) $\forall A, B, C \in F(X)$, if $A \subset B \subset C$, then $s(A, B) \geq s(A, C)$ and $s(B, C) \geq s(A, C)$,

where $F(X)$ is a fuzzy set and $P(X)$ is a numeric set

Burillo and Bustince proposed an axiomatic definition of IFSs, which was considered by taking into account fuzzy set consideration. Consider Gaussian type IFS membership function in Fig. 1.

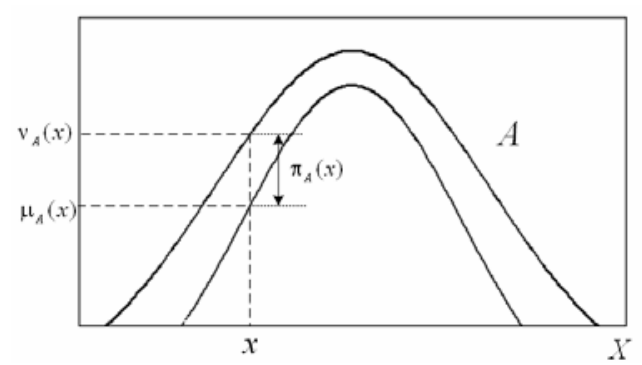

Fig. 1 Gaussian type IFS Membership Function.

Definition 2.4 [16] A real function $I: \operatorname{IFS}(X) \rightarrow R^{+}$is called an entropy on IFS(X) if $I$ has the following properties:

(IP1) $I(A)=0$, if and only if $A$ is a fuzzy set,

(IP2) $\quad I(A)=\operatorname{Cardinal}(X)=N$ if and only if $\mu_{A}(x)=v_{A}(x)=0$ for all $x \in X$,

(IP3) $I(A)=I\left(A^{C}\right)$ for all $A \in \operatorname{IFSs}(X)$,

(IP4) if $A \pi B$, then $I(A) \geq I(B)$.

Where $A \pi B$ denotes that $\mu_{A}(x) \leq \mu_{B}(x)$ and $v_{A}(x) \leq v_{B}(x)$ for all $x \in X$, which means that IFS $B$ has less hesitancy than IFS $A . \mu_{A}, v_{A}$, and $\pi_{A}$ are degree of membership, non-membership, and hesistancy of $x$ to $A$, that is, $\pi_{A}(x)=1-\mu_{A}(x)-v_{A}(x)$.

Next, similarity measure between IFSs has been introduced by Dengfeng and Chuntian, it has similar formulation with Definition 2.3 [17].

Definition 2.5 A mapping $S: \operatorname{IFS}(X) \times \operatorname{IFS}(X) \rightarrow[0,1]$. IFSs(X) denotes the set of all IFSs in $X=\left\{x_{1}, x_{2}, \Lambda, x_{n}\right\}$. $S(A, B)$ is said to be the degree of similarity between $A \in \operatorname{IFS}(X)$ and $B \in \operatorname{IFS}(X)$, if $S(A, B)$ satisfies the properties of conditions:

(P1) $S(A, B) \in[0,1]$,

(P2) $S(A, B)=1 \Leftrightarrow A=B$,

(P3) $S(A, B)=S(B, A)$,

(P4) $\quad S(A, C) \leq S(A, B)$ and $\quad S(A, C) \leq S(B, C) \quad$ if $A \subset B \subset C, C \in \operatorname{IFS}(X)$,

(P5) $S(A, B)=0 \Leftrightarrow A=\Phi$ and $B=\bar{A}$, or, $A=\bar{B}$ and $B=\Phi$,

where $\Phi$ means that information of IFS is very clear, $\mu_{A}=0$ and $v_{A}=1 . \bar{A}$ denotes the IFS complement of A.

With the definition of fuzzy entropy and similarity measure, various entropy and similarity measure have been introduced [911]. The usefulness also verified through previous results. Comparison between IFSs similarity measure was done by Y. Li, D.L. Olson, and Z. Qin [18]. They have compared conventional similarity measures. Furthermore, another fuzzy entropy for IFSs has been also defined by Hung and Yang [19]. In their definition, IP2 and IP4 are different from that of their properties. Difference of two definitions has their own characteristics.

Next, entropy and similarity measure between IFSs has been derived based on Definition 2.4 and 2. Results are somewhat similar with that of fuzzy set.

\section{Fuzzy Entropy and Similarity Measure on IFSs}

Fuzzy entropy design for IFSs has been reported by fewer researchers than similarity measure. Burillo and Bustince in 1996 and Szmidt and Kacprzyk in 2001 have proposed the fuzzy entropy of IFSs $[16,20]$. They allow us to measure the degree of intuitionism an IFS, and nonprobabilistic type entropy measure with a geometric interpretation of IFSs.

\subsection{Fizzy Entropy for IFSs with Distance Measure}

IFSs entropy Definition 2.4 means that fuzzy entropy of IFSs is the area of hesistancy, that is, $\pi_{A}(x)=1-\mu_{A}(x)-v_{A}(x)$. Result was shown in [16]. Now we propose fuzzy entropy with well known Hamming distance, following theorem consider modified assumption about IP1. 
Another fuzzy entropy on IFSs has been also defined by Hung and Yang [19]. In their definition, IP2 and IP4 are different from that of their properties. Difference of two definitions has their own characteristics. In Definition 2.4, IP1 express that all fuzzy set has entropy zero property. However, it is unmatched to the actual consideration, because of the property (E1) in Definition 2.2. Hence, it is required to change the assumption into:

(IP1) $I(A)=0$, if and only if $A$ is a crisp set,

then more general fuzzy entropy of IFSs can be possible as next theorem.

Theorem 3.1 Following equation satisfies a fuzzy entropy on IFS(X).

$$
E_{L}\left(A, A_{\text {near }}\right)=\frac{1}{N} \sum_{i=1}^{N} \pi_{A}\left(x_{i}\right)+d\left(\mu_{A}\left(x_{i}\right), \mu_{A_{\text {near }}}\right) !
$$

Where subscript "near" means close crisp set to set $A$,

in this theorem near $=0.5$ is considered, then $\mu_{A_{\text {near }}}=1$ when $\mu_{A} \geq 0.5$ and $\mu_{A_{\text {near }}}=0$ when $\mu_{A} \leq 0.5$.

Proof: For normal fuzzy membership function, (1) represent $d\left(\mu_{A}\left(x_{i}\right), \mu_{A_{\text {near }}}\right)=0$. Hence, it is the same result of [16]. For unnormal fuzzy membership function, (1) is clear because $A_{\text {near }}$ satisfies $A$ itself. Therefore, (IP1)' is satisfied. For (IP2), $d\left(\mu_{A}\left(x_{i}\right), \mu_{A_{\text {near }}}\right)=0 \quad$ for $\quad \mu_{A}(x)=0 \quad$ and $v_{A}(x)=0$. It is also natural, $I(A)=\operatorname{Cardinal}(X)=N$. (IP3) is clear by the definition. Finally, if $A \pi B$, then $\frac{1}{N} \sum_{i=1}^{N} \pi_{A}\left(x_{i}\right) \geq \frac{1}{N} \sum_{i=1}^{N} \pi_{A}\left(x_{i}\right)$ and $d\left(\mu_{A}\left(x_{i}\right), \mu_{A_{\text {near }}}\right)$. $\geq d\left(\mu_{A}\left(x_{i}\right), \mu_{A_{\text {near }}}\right)$

Theorem 3.1 considers for more flexible fuzzy membership function as well as normal fuzzy membership function. Hence, (1) is useful as a fuzzy entropy for IFSs. Fuzzy entropy has to be carried out between IFS and corresponding numeric data or set. Where as, similarity measures are designed by comparison between IFSs.

\subsection{Similarity Measure for IFSs with Distance Measure}

Similarity measures for IFSs contained counter-intuitive cases [18]. Especially, condition P2 is very strict to overcome, because similarity measure based on the difference calculation between two IFSs. For example, Hong and Kim showed similarity measure as follows [18];

$S_{H}(A, B)=1-\frac{\sum_{i=1}^{n}\left(\left|t_{A}\left(x_{i}\right)-t_{B}\left(x_{i}\right)\right|\right.}{\left.+\left|f_{A}\left(x_{i}\right)-f_{B}\left(x_{i}\right)\right|\right)}$

Equation (2) has counter-intuitive case for P2, and the result can be shown in [18]. Other conventional similarity measure showed almost same results. Here novel similarity measure between IFSs is considered as follows.

Theorem 3.2 Following equation satisfies a similarity measure on $\operatorname{IFS}(\mathrm{X})$.

$$
S_{L}(A, B)=1-E_{L}(A, B) .
$$

Where $E_{L}(A, B)$ is the same formulation of (1), and $A_{\text {near }}$ is replaced into $B$.

Proof: From (P1) to (P3), it is clear from (3) itself. And $d\left(\mu_{A}\left(x_{i}\right), \mu_{C}\left(x_{i}\right)\right)$ and $d\left(\mu_{A}\left(x_{i}\right), \mu_{C}\left(x_{i}\right)\right)$ are greater than $d\left(\mu_{A}\left(x_{i}\right), \mu_{B}\left(x_{i}\right)\right)$ and $d\left(\mu_{B}\left(x_{i}\right), \mu_{C}\left(x_{i}\right)\right)$, respectively. Hence, (P4) is satisfied. Finally, $A=\Phi$ and $B=\bar{A}$, or, $A=\bar{B}$ and $B=\Phi$, IFSs $A$ and $B$ are complementary each other., therefore (P5) is also satisfied.

By the above proof, (3) is considered as the similarity measure for IFSs

\subsection{Discussion on Fuzzy Entropy of IFSs}

Since the fuzzy set was introduced by Zadeh, many new approaches and theories treating imprecision and uncertainty have been proposed, such as the interval-valued fuzzy sets. Among these theories, a extension of the classic fuzzy set is intuitionistic fuzzy set theory, which was introduced by Atanassov [12,13]. Since then, many researchers have investigated this topic and obtained some meaningful conclusions such as implication of intuitionistic fuzzy sets, generalization of intuitionistic fuzzy rough approximation operators, and multi-criteria decision-making methods based on intuitionistic fuzzy sets.

Fuzzy entropy and similarity measure represent dual meaning each other, that is, dissimilarity and similarity between considering two sets. In the previous result, their relation was showed and discussed about each characteristics [9]. Equations (1) and (3) also represent same results. Similarity measure (3) shows little difference with fuzzy set. However, fuzzy entropy contains some debate from definition itself. In Definition 2.4, fuzzy entropy was defined by the hesistancy area, there it was not necessary corresponding numerical data. Basically, fuzzy set contains the uncertainty in itself, which is evaluated by the entropy measure. Hence, additional term between $\mu_{A}\left(x_{i}\right)$ and $\mu_{A_{\text {near }}}$ is considered, which make possible to consider nonnormal intuitionistic fuzzy membership function. Furthermore, relation between fuzzy entropy and similarity measure shows similar result compared to our previous result.

\section{Conclusions}

Fuzzy entropy and similarity measure realization on IFSs have been done. With the help of fuzzy entropy and similarity measure of fuzzy set, analysis on IFSs also carried out. 
Usefulness of proposed measures is proved by evaluating the definitions. Relation between fuzzy entropy and similarity measure are also discussed. By discussing the relation, it was verified that one measure is derived from another measure, hence two measures satisfy dual concept for data analysis.

\section{Acknowledgment}

This paper was supported by the second stage of Brain Korea 21 Projects. This work was also supported by Priority Research Centers Program through the National Research Foundation of Korea(NRF) funded by the Ministry of Education, Science and Technology(20100020163)

\section{References}

[1] Zadeh LA. "Fuzzy sets and systems", Proc Symp on Systems Theory, Polytechnic Institute of Brooklyn, New York; 1965. pp 29-37

[2] N.R. Pal and S.K. Pal, "Object-background segmentation using new definitions of entropy”, IEEE Proc,. Vol. 36, 284 295, 1989.

[3] B. Kosko, Neural Networks and Fuzzy Systems, Prentice-Hall, Englewood Cliffs, NJ, 1992.

[4] Liu Xuecheng, "Entropy, distance measure and similarity measure of fuzzy sets and their relations", Fuzzy Sets and Systems, Vol. 52, 305-318, 1992.

[5] D. Bhandari and N.R. Pal, "Some new information measure of fuzzy sets”, Inform. Sci. Vol. 67, 209-228, 1993.

[6] DeLuca and S. Termini, "A Definition of nonprobabilistic entropy in the setting of fuzzy entropy", J. General Systems, vol. 5, 1972, pp. 301-312.

[7] C.H. Hsieh and S.H. Chen, "Similarity of generalized fuzzy numbers with graded mean integration representation", Proc. 8th Int. Fuzzy Systems Association World Congr., Vol 2, 1999, pp. 551-555.

[8] S.J. Chen and S.M. Chen, "Fuzzy risk analysis based on similarity measures of generalized fuzzy numbers", IEEE Trans. on Fuzzy Systems, Vol. 11, no. 1, 2003, pp. 45-56.

[9] S.H. Lee, W. Pedrycz, and Gyoyong Sohn, "Design of Similarity and Dissimilarity Measures for Fuzzy Sets on the Basis of Distance Measure", International Journal of Fuzzy Systems, vol. 11, 2009, pp. 67-72.

[10] S.H. Lee, K.H.Ryu, G.Y. Sohn, "Study on Entropy and Similarity Measure for Fuzzy Set”, IEICE Trans. Inf. \& Syst., vol. E92-D, Sep. 2009, pp. 1783-1786.

[11] S.H. Lee, S. J. Kim, N. Y. Jang, "Design of Fuzzy Entropy for Non Convex Membership Function”, CCIS, vol. 15, 2008, pp. 55-60, doi:10.1007/978-3-540-85930-7.

[12] Atanassov K. "Intuitionistic fuzzy sets", Fuzzy Set and Syst . vol. 20, pp. 87-96, 1986.

[13]Atanassov K. Intuitionistic fuzzy sets: Theory and applications, Heidelberg: Physica-Verlag; 1999.

[14] Atanassov K. New operations defined over the intuitionistic fuzzy sets. Fuzzy Set and Sys., 1994;61:137-142.

[15] W.L. Gau, Buehrer, D.J, "Vague sets", IEEE Trans. Syst. Man Cybernet. Vol 23, No. 2, 1993, pp. 610-614.

[16] P. Burillo, H. Bustince, "Entropy on intuitionistic fuzzy sets and on interval-valued fuzzy sets", Fuzzy Sets and Systems, Vol. 78, 1996, pp. 305-316.

[17] Dengfeng, L., Chuntian, C., "New similarity measure of intiotionistic fuzzy sets and application to pattern recognitions", Pattern Recognotion Lett., vol. 23, 2002, pp. 221-225.

[18] Yanhong Li, David L. Olson, Zheng Qin, "Similarity measures between intuitionistic fuzzy (vague) set: A comparative analysis", Pattern Recognition Lett., vol 28, 2007, pp. 278-285.

[19] W. L. Hung, M. S. Yang, "Fuzzy entropy on inttuitionistic fuzzy sets", International Journal of Intelligent Systems, vol 21, 2006, pp. 443-451.

[20]E. Szmidt, J. Kacprzyk, "Entropy for intuitionistic fuzzy sets", Fuzzy Sets and Systems, vol 118, 2001, pp. 467-477.

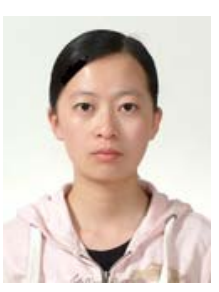

\section{Hong-mei Wang}

received the B.S degree in Automation Control Engineering at Qingdao University, China, in 2006. Now she is in School of Mechatronics Engineering, Changwon National University, Korea, for M.S degree. Her research interests include in the area of designing system applied for wireless communication modem and various systems required advanced digital signal processing

E-mail : iwanghongmei99@163.com

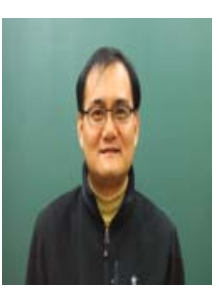

\section{Sanghyuk Lee}

received the B.S. in EE from Chungbuk National University, in 1988, M.S. and Ph.D. degrees in EE from Seoul National University, in 1991 and 1998, respectively. Dr. Lee served as a Research Fellow from 1996 to 1999 in HOW Company. Currently, he has been with the Institute for Information and Electronics Research in Inha University as a research professor since 2010. His research interests include fuzzy theory, game theory, controller design for linear and nonlinear systems.

E-mail : leehyuk@inha.ac.kr 